\title{
Design and Analysis of Multichannel Slotted ALOHA for Machine-to-Machine Communication
}

\author{
Chih-Hua Chang* and Ronald Y. Chang ${ }^{\dagger}$ \\ ${ }^{*}$ School of Electrical and Computer Engineering, Purdue University, USA \\ ${ }^{\dagger}$ Research Center for Information Technology Innovation, Academia Sinica, Taiwan \\ Email: chang377@purdue.edu, rchang@citi.sinica.edu.tw
}

\begin{abstract}
In machine-to-machine (M2M) communication, a massive number of machine devices may transmit simultaneously in response to an event occurring in the system. Supporting massive device transmission while maintaining low congestion and low access delay is a challenging problem. This paper proposes a new transmission control scheme based on slotted ALOHA, with a practical consideration of partial information available at the data aggregator about the system. The proposed approximate maximum likelihood estimation ALOHA (AMLEALOHA) scheme incorporates an approximate ML estimation of the (unknown) number of active machines in the system. We apply the drift analysis to show the stability of the proposed control scheme. Simulation results demonstrate that the proposed AMLE-ALOHA outperforms an existing scheme in terms of the access delay and reaction time under bursty traffic with the same partial information, and compares favorably to the optimal control scheme with oracle knowledge of the number of active machines in the system.
\end{abstract}

\section{INTRODUCTION}

Machine-to-machine (M2M) communication has found many emerging applications and attracted significant research interest recently. A typical M2M communication scenario is where a data aggregator collects data generated by a large number of machine devices (e.g., sensors). In fact, massive machine communications are envisioned for future wireless communications [1]. When there is a massive number of machines transmitting data to the aggregator concurrently in a single-hop or direct-communication manner [2], a good congestion control scheme is needed to mitigate traffic congestion due to intense machine access in M2M [3].

Many congestion control schemes for M2M have been proposed, some from the 3GPP standardization effort. Access Class Barring (ACB), which was proposed as an overload control mechanism dividing machines into several access classes and performing the access barring check before establishing a connection [4], has been adapted to M2M communications [5], [6]. An enhancement of ACB, termed Extended Access Barring (EAB), was later proposed and discussed in standards to address the overload in the random access channel when a large number of machines need to access the network in a short burst [5]. In [7], a new control algorithm was proposed and used to adjust the parameter of the ACB mechanism. The ACB

This work was supported in part by the Ministry of Science and Technology, Taiwan, under Grants MOST 103-2221-E-001-027 and MOST 104-2628-E001-002-MY2. mechanism and its variants are based on the well-developed and widely used slotted ALOHA (S-ALOHA) protocol [8]. It is well-known that uncontrolled S-ALOHA scheme is unstable such that the number of backlogged devices may grow unbounded over time with probability one [9]. In order to implement the S-ALOHA scheme with efficiency and stability, it is essential to estimate the number of active machines (i.e., machines that have data packets to transmit to the aggregator as a result of an event having taken place) and optimize the transmission probabilities of these active machines. The Pseudo Bayesian ALOHA (PB-ALOHA) scheme [10], [11] estimates the number of active machines based on the access outcome in the previous time slot, but cannot quickly adjust the transmission probability to test the active machines under highly bursty traffic [12].

In this paper, we consider an M2M communication system with a massive number of machines directly communicating with the data aggregator. We propose a new transmission probability control scheme for multichannel S-ALOHA. The proposed scheme is based on an approximate maximum likelihood estimation of the number of active machines, thus termed approximate maximum likelihood estimation ALOHA (AMLE-ALOHA) scheme. We analytically study the stability properties of AMLE-ALOHA by performing the drift analysis. Numerical results demonstrate that the proposed AMLEALOHA scheme yields reduced access delay at high packet arrival rates and reduced reaction time under bursty traffic as compared to the PB-ALOHA scheme.

The outline of this paper is as follows. Sec. II presents the system model. Sec. III describes benchmark control schemes. Sec. IV describes the proposed AMLE-ALOHA scheme. Sec. $\mathrm{V}$ shows the stability of AMLE-ALOHA. Sec. VI presents simulation results. Finally, Sec. VII concludes this work.

\section{SySTEM MODEL}

We consider a cellular network based M2M communication system as shown in Fig. 1. The system consists of a data aggregator or base station (BS) and many machine devices which will upload data to the BS. There are $K$ synchronous and noninterfering channels available for use for data uploading. We consider a slotted transmission system, where any packet transmission starts at the beginning of a time slot and finishes at the end of the same time slot. 


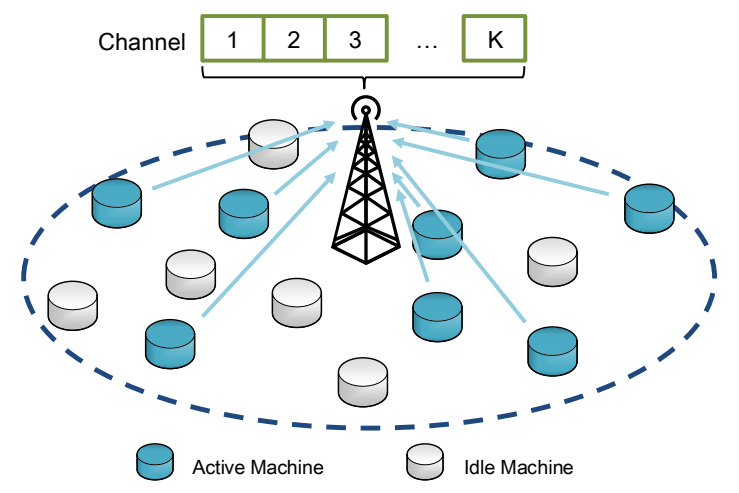

Fig. 1. A cellular network-based M2M communication system.

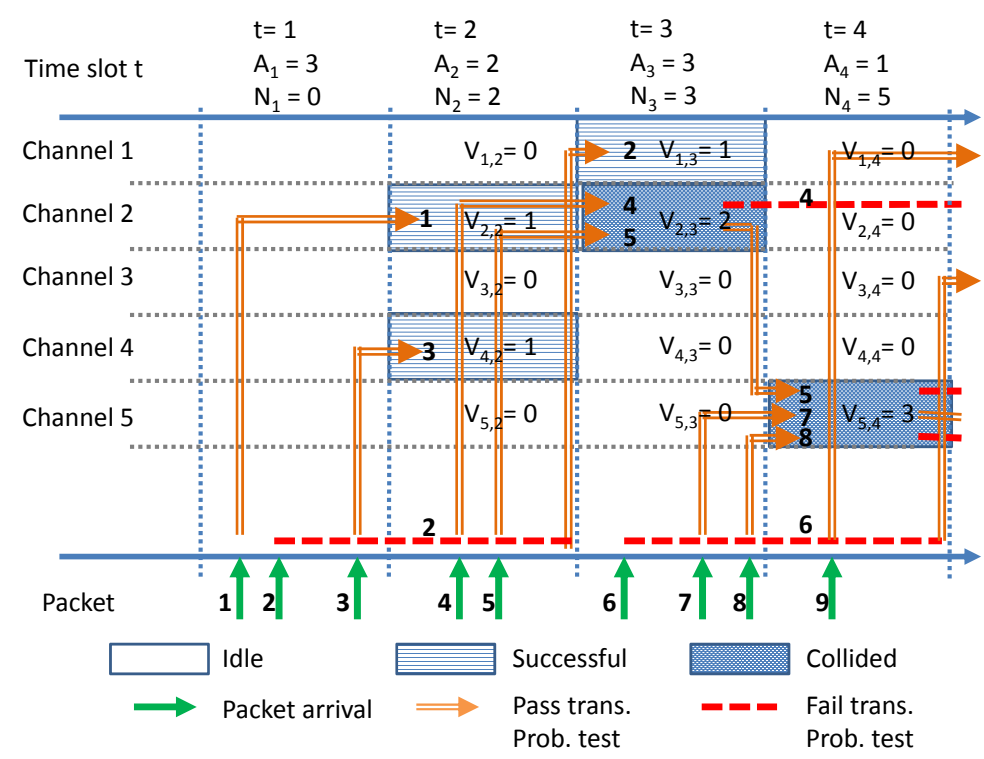

Fig. 2. Dynamic packet arrivals and transmissions. Packet 1 arrived in time slot 1 and was transmitted successfully on channel 2 in time slot 2; packet 2 arrived in time slot 1 and was put on hold (due to failing the "transmission probability test") until time slot 3 where it was transmitted successfully on channel 1; packets 4 and 5 arrived in time slot 2 and both were transmitted unsuccessfully on channel 3 in time slot 3 due to collision, and both attempted transmission again in time slot 4 with different outcomes; etc.

Packet arrival model: The number of packet arrivals $A_{t}$ in time slot $t$ follows i.i.d. probability distribution $f\left(A_{t}\right)$ for all $t \in \mathbb{Z}^{+}=\{1,2,3, \ldots\}$, where $E\left[A_{t}\right]=\lambda$ and $E\left[A_{t}^{2}\right]<\infty$. Each arrival is independently routed to a machine for transmission to the BS. Specifically, $A_{t}$ packet arrivals in time slot $t$ will be routed to $A_{t}$ machines each attempting to transmit a packet to the $\mathrm{BS}$ in time slot $t+1$. The assignment of machines for packet transmissions is unspecified.

Transmission and multiaccess mechanism: A slotted ALOHA random-access protocol with a packet transmission probability control scheme is employed. The BS broadcasts a persistence probability value $p_{t}$ to the system at the end of each time slot $t-1$. Machines with packets to send in time slot $t$ are called active machines and otherwise called idle machines in time slot $t$. Active machine $i\left(i=1, \ldots, N_{t}\right.$, where $N_{t}$ is the number of active machines in time slot $t$ ) will take a "transmission probability test," i.e., generate a uniform random variable $q_{i, t} \in[0,1]$ such that: if $q_{i, t}>p_{t}$, machine $i$ will not transmit in time slot $t$ and will attempt transmission again in time slot $t+1$ by following the same procedure (i.e., generating $q_{i, t+1} \in[0,1]$ and comparing $q_{i, t+1}$ and $p_{t+1}$ ), and otherwise $\left(q_{i, t} \leq p_{t}\right)$ machine $i$ will transmit one packet in time slot $t$ using a channel randomly and equiprobably selected from the $K$ channels. In any time slot, if a channel is used exclusively by one machine, this machine's transmission is assumed successful; otherwise, all transmissions on this channel fail due to collision. In case of collisions, the machines failing transmissions in time slot $t$ will all attempt transmission again in time slot $t+1$. Thus, the number of active machines in time slot $t+1\left(N_{t+1}\right)$ is the sum of the number of machines that could not complete transmission in all time slots prior to time slot $t+1$ and the number of packet arrivals in time slot $t$ $\left(A_{t}\right)$. We call the active machines in time slot $t$ the backlogged machines in time slot $t$. After successfully transmitting a 
packet, an active machine will become idle since the next time slot, until a packet is routed to it again. An example of dynamic packet arrivals and transmissions is illustrated in Fig. 2.

Objective: We consider (as in [11], [13]) that it is difficult for the BS to know $N_{t}$ but easier for the BS to observe the states of the $K$ channels, i.e., i) idle $\left(v_{k, t}=0\right)$, ii) successful $\left(v_{k, t}=1\right)$, or iii) collided $\left(v_{k, t} \geq 2\right)$, where $v_{k, t}$ is the number of machines attempting to use the $k$-th channel in time slot $t$. Note that in iii) the BS only knows the contention outcome (i.e., collided) but not the precise number of machines involved in a collision. Given this partial information about the system, the BS can dynamically adjust the persistence probability $p_{t}$ according to the packet arrival rate and the number of available channels. Our objective is to propose a new packet transmission probability control scheme that improves the throughput and/or delay performance of the existing scheme with same partial information, and that compares favorably to the optimal control scheme with full information (i.e., $N_{t}$ ).

\section{Benchmark Control Schemes}

\section{A. Optimal Control Scheme}

The optimal persistence probability $p_{t}^{*}$ is one that yields the minimum number of active or backlogged machines in time slot $t+1$.

Theorem 1 [13]. For i.i.d. arrival flow $A_{t}$, where $E\left[A_{t}\right]=\lambda$ and $E\left[A_{t}^{2}\right]<\infty$, the persistence probability that maintains the minimum number of backlogged machines in the system, i.e., $E\left[N_{t+1} \mid p_{t}^{*}\right] \leq E\left[N_{t+1} \mid p_{t}\right]$ for all $t \in \mathbb{Z}^{+}$and $p_{t} \in[0,1]$, is given by $p_{t}^{*}=\frac{K}{N_{t}}$.

Note that the optimal control scheme requires knowledge of $N_{t}$ at the BS, which may not be available in practical distributed systems [11]. The following method is based only on the information of the states of the $K$ channels (idle, successful, or collided) and the previous control results $\left(p_{t-1}\right)$ in designing $p_{t}$.

\section{B. Pseudo Bayesian ALOHA (PB-ALOHA) Scheme}

Suppose that there are $m$ machines out of $N_{t}$ active machines that are transmitting in time slot $t$, and machine $i$ $(i=1,2, \ldots, m)$ uses channel $w_{i, t}$ for transmission. Then, we can express $v_{k, t}=\sum_{i=1}^{m} I\left(w_{i, t}=k\right)$, where $I(\cdot)$ is the indicator function. Define the channel state triplet $\left(K_{d, t}, K_{s, t}, K_{c, t}\right)$, where $K_{d, t}=\sum_{k=1}^{K} I\left(v_{k, t}=0\right), K_{s, t}=\sum_{k=1}^{K} I\left(v_{k, t}=1\right)$, and $K_{c, t}=\sum_{k=1}^{K} I\left(v_{k, t} \geq 2\right)$ are the numbers of idle, successful, and collided channels in time slot $t$, respectively. Clearly, $K_{d, t}+K_{s, t}+K_{c, t}=K$. Then, the PB-ALOHA control scheme is given as follows [11], [13]:

$$
p_{t}^{\mathrm{PB}}=\min \left\{1, \frac{K}{N_{t}^{\mathrm{PB}}}\right\}
$$

where

$$
N_{t}^{\mathrm{PB}}=\max \left\{1, N_{t-1}^{\mathrm{PB}}-K_{d, t}-K_{s, t}+\frac{2 K_{c, t}}{e-2}\right\} .
$$

\section{The Proposed Approximate MAXimum LIKELIHOOD ESTIMATION ALOHA (AMLE-ALOHA) SCHEME}

Our method is based on an approximate ML estimation of the number of active machines $N_{t}$ given $\left(K_{d, t}, K_{s, t}, K_{c, t}\right)$ and $p_{t-1}$. First, the conditional probability that the BS observes the channel state triplet $\left(K_{d, t}, K_{s, t}, K_{c, t}\right)$ given $N_{t}=n$ and $p_{t-1}$ can be expressed as

$$
\begin{aligned}
& \operatorname{Pr}\left(K_{d, t}, K_{s, t}, K_{c, t} \mid N_{t}=n, p_{t-1}\right) \\
&=\sum_{m=0}^{n} \Phi\left(K_{d, t}, K_{s, t}, K_{c, t} \mid m\right) \cdot \Omega(m \mid n)
\end{aligned}
$$

where

$$
\Omega(m \mid n)=\left(\begin{array}{c}
n \\
m
\end{array}\right) p_{t-1}^{m}\left(1-p_{t-1}\right)^{n-m}
$$

is the probability that $m$ out of $n$ active machines are transmitting in time slot $t$, and $\Phi\left(K_{d, t}, K_{s, t}, K_{c, t} \mid m\right)$ is the probability that the BS observes channel state triplet $\left(K_{d, t}, K_{s, t}, K_{c, t}\right)$ given that $m$ out of $n$ active machines are transmitting, as derived as follows.

Theorem 2.

$$
\begin{aligned}
& \Phi\left(K_{d, t}=d, K_{s, t}=s, K_{c, t}=c \mid m\right) \\
& = \begin{cases}\frac{s !}{K^{m}} \sum_{l=0}^{c} l !\left(\begin{array}{c}
m \\
s, l
\end{array}\right) \sum_{j=0}^{c-l}\left(\begin{array}{c}
K \\
d, s, l, j
\end{array}\right)(-1)^{l+j}(c-l-j)^{m-s-l}, \\
0, & \text { if } m \geq K_{s, t}+K_{c, t} \\
0, & \text { if } m<K_{s, t}+K_{c, t}\end{cases}
\end{aligned}
$$

Proof. The case of $m<K_{s, t}+K_{c, t}$ is straightforward. Thus, we show the case of $m \geq K_{s, t}+K_{c, t}$. By definition, we have

$$
\begin{aligned}
& \Phi\left(K_{d, t}=d, K_{s, t}=s, K_{c, t}=c \mid m\right) \\
& =\left(\begin{array}{c}
K \\
d, s
\end{array}\right)\left(\begin{array}{c}
m \\
s
\end{array}\right)\left(\frac{s}{K}\right)^{s} \Phi(0, s, 0 \mid s)\left(\frac{c}{K}\right)^{m-s} \Phi(0,0, c \mid m-s)
\end{aligned}
$$

where

$$
\Phi(0, s, 0 \mid s)=\frac{s !}{s^{s}} .
$$

That is, given the channel state triplet $(d, s, c),\left(\frac{s}{K}\right)^{s} \Phi(0, s, 0$ | $s)$ is the probability that $s$ selected machine have successful transmission, and $\left(\frac{c}{K}\right)^{m-s} \Phi(0,0, c \mid m-s)$ is the probability that the remaining $m-s$ machines have collided transmission. To derive $\Phi(0,0, c \mid m-s)$, we consider, without loss of generality, a scenario where $a$ machines each choose a transmission channel among a set of $b$ channels $\mathcal{B} \triangleq\{1,2, \ldots, b\}$. Let $\mathbf{w}_{t} \triangleq\left[w_{1, t}, \ldots, w_{a, t}\right]$ denote the channels used by these $a$ machines, and $\mathcal{S} \triangleq\left\{\mathbf{w}_{t}: v_{k, t} \geq 1, \forall k \in \mathcal{B}\right\}$ represent the combinations that $a$ machines use all the $b$ channels without any idle channel, where $\operatorname{Pr}(\mathcal{S})=\Theta(b, a)$ as given by [14]

$$
\Theta(b, a)=\left\{\begin{array}{ll}
\sum_{j=0}^{b}(-1)^{j}\left(\begin{array}{l}
b \\
j
\end{array}\right)\left(\frac{b-j}{b}\right)^{a}, & a \geq b \\
0, & a<b
\end{array} .\right.
$$

Furthermore, let $\mathcal{A}_{j} \triangleq\left\{\mathbf{w}_{t}: v_{j, t}=1, v_{k, t} \geq 1, \forall k \in \mathcal{B} \backslash\right.$ $\{j\}\}$ represent the combinations that $a$ machines use all the 
$b$ channels without any idle channel and at least one machine successfully transmits (using channel $j$ ). Thus, the intersection $\bigcap_{j \in \mathcal{J} \subseteq \mathcal{B},|\mathcal{J}|=l} \mathcal{A}_{j}=\left\{\mathbf{w}_{t}: v_{j, t}=1, \forall j \in \mathcal{J} \subseteq \mathcal{B},|\mathcal{J}|=\right.$ $\left.l, v_{k, t} \geq 1, \forall k \in \mathcal{B} \backslash \mathcal{J}\right\}$ represents the combinations that $a$ machines use all the $b$ channels without any idle channel and at least $l$ machines successfully transmit (using the set of channels $\mathcal{J}$, where $|\mathcal{J}|=l$ ). By definition, we have

$\operatorname{Pr}\left(\bigcap_{j \in \mathcal{J} \subseteq \mathcal{B},|\mathcal{J}|=l} \mathcal{A}_{j}\right)=\left(\begin{array}{l}a \\ l\end{array}\right) \frac{l !}{b^{l}}\left(\frac{b-l}{b}\right)^{a-l} \Theta(b-l, a-l)$.

Then, we apply the inclusion-exclusion principle to find the probability that all $b$ channels are collided given that $a$ machines are transmitting:

$$
\begin{aligned}
& \Phi(0,0, b \mid a)=\operatorname{Pr}\left(\mathcal{S}-\bigcup_{j \in \mathcal{B}} \mathcal{A}_{j}\right) \\
& =\operatorname{Pr}(\mathcal{S})+\sum_{\mathcal{J} \subseteq \mathcal{B}, \mathcal{J} \neq \emptyset}(-1)^{|\mathcal{J}|} \operatorname{Pr}\left(\bigcap_{j \in \mathcal{J}} \mathcal{A}_{j}\right) \\
& =\operatorname{Pr}(\mathcal{S})+\sum_{l=1}^{b} \sum_{|\mathcal{J}|=l, \mathcal{J} \subseteq \mathcal{B}}(-1)^{|\mathcal{J}|} \operatorname{Pr}\left(\bigcap_{j \in \mathcal{J}} \mathcal{A}_{j}\right) \\
& =\Theta(b, a)+\sum_{l=1}^{b}\left(\begin{array}{l}
b \\
l
\end{array}\right)(-1)^{l} \operatorname{Pr}\left(\bigcap_{j \in \mathcal{J} \subseteq \mathcal{B},|\mathcal{J}|=l} \mathcal{A}_{j}\right) \\
& =\sum_{l=0}^{b}(-1)^{l}\left(\begin{array}{l}
a \\
l
\end{array}\right)\left(\begin{array}{l}
b \\
l
\end{array}\right) \frac{l !}{b^{l}}\left(\frac{b-l}{b}\right)^{a-l} \Theta(b-l, a-l)
\end{aligned}
$$

where $a \geq b$. Substituting $a=m-s$ and $b=c$ in (10) and (8) yields

$$
\begin{aligned}
& \Phi(0,0, c \mid m-s) \\
& =\sum_{l=0}^{c} \frac{(-1)^{l} l !}{c^{m-s}}\left(\begin{array}{c}
m-s \\
l
\end{array}\right)\left(\begin{array}{l}
c \\
l
\end{array}\right) \sum_{j=0}^{c-l}\left(\begin{array}{c}
c-l \\
j
\end{array}\right)(-1)^{j}(c-l-j)^{m-s-l} \\
& =\sum_{l=0}^{c} \frac{l !}{c^{m-s}}\left(\begin{array}{c}
m-s \\
l
\end{array}\right) \sum_{j=0}^{c-l}\left(\begin{array}{c}
c \\
l
\end{array}\right)\left(\begin{array}{c}
c-l \\
j
\end{array}\right)(-1)^{l+j}(c-l-j)^{m-s-l} \\
& =\sum_{l=0}^{c} \frac{l !}{c^{m-s}}\left(\begin{array}{c}
m-s \\
l
\end{array}\right) \sum_{j=0}^{c-l}\left(\begin{array}{c}
c \\
l, j
\end{array}\right)(-1)^{l+j}(c-l-j)^{m-s-l}
\end{aligned}
$$

where the last equality holds since $\left(\begin{array}{c}c \\ l\end{array}\right)\left(\begin{array}{c}c-l \\ j\end{array}\right)=\left(\begin{array}{c}c \\ l, j\end{array}\right)$ under the condition $m-s \geq c$ or equivalently $m \geq K_{s, t}+K_{c, t}$. Combining (6), (7), and (11) leads to

$$
\begin{aligned}
& \Phi(d, s, c \mid m) \\
& =\frac{s !}{K^{m}}\left(\begin{array}{c}
K \\
d, s
\end{array}\right)\left(\begin{array}{c}
m \\
s
\end{array}\right) \sum_{l=0}^{c} l !\left(\begin{array}{c}
m-s \\
l
\end{array}\right) \sum_{j=0}^{c-l}\left(\begin{array}{c}
c \\
l, j
\end{array}\right)(-1)^{l+j}(c-l-j)^{m-s-l} \\
& =\frac{s !}{K^{m}} \sum_{l=0}^{c} l !\left(\begin{array}{c}
m \\
s
\end{array}\right)\left(\begin{array}{c}
m-s \\
l
\end{array}\right) \sum_{j=0}^{c-l}\left(\begin{array}{c}
K \\
d, s
\end{array}\right)\left(\begin{array}{c}
c \\
l, j
\end{array}\right)(-1)^{l+j}(c-l-j)^{m-s-l} \\
& =\frac{s !}{K^{m}} \sum_{l=0}^{c} l !\left(\begin{array}{c}
m \\
s, l
\end{array}\right) \sum_{j=0}^{c-l}\left(\begin{array}{c}
K \\
d, s, l, j
\end{array}\right)(-1)^{l+j}(c-l-j)^{m-s-l}
\end{aligned}
$$

where the last equality holds since $\left(\begin{array}{c}K \\ d, s\end{array}\right)\left(\begin{array}{c}c \\ l, j\end{array}\right)=$ $\left(\begin{array}{c}K \\ d, s\end{array}\right)\left(\begin{array}{c}K-d-s \\ l, j\end{array}\right)=\left(\begin{array}{c}K \\ d, s, l, j\end{array}\right)$.

With the expressions in (3), (4), and (5), we propose a control scheme based on the ML estimation of $N_{t}$ as follows:

$$
p_{t}^{\mathrm{ML}}=\min \left\{1, \frac{K}{N_{t}^{\mathrm{ML}}}\right\}
$$

where

$$
N_{t}^{\mathrm{ML}}=\underset{n \in \mathbb{Z}^{+}}{\arg \max } \operatorname{Pr}\left(K_{d, t}, K_{s, t}, K_{c, t} \mid N_{t}=n, p_{t-1}^{\mathrm{ML}}\right)
$$

is the ML estimator of $N_{t}$ in time slot $t$. However, applying the ML estimation according to (14) in each time slot is computationally expensive. Thus, it is worthwhile to consider an approximate estimator. Consider an approximation of the distribution $\Omega(m \mid n)$ in (4) by another distribution $\Lambda(m \mid n)$, where $\Lambda(m \mid n)=1$ for $m=n p_{t-1}$ and $\Lambda(m \mid n)=0$ otherwise. Therefore, (14) becomes

$$
\begin{aligned}
N_{t}^{\mathrm{ML}} & =\underset{n \in \mathbb{Z}^{+}}{\arg \max } \operatorname{Pr}\left(K_{d, t}, K_{s, t}, K_{c, t} \mid N_{t}=n, p_{t-1}^{\mathrm{ML}}\right) \\
& =\underset{n \in \mathbb{Z}^{+}}{\arg \max } \sum_{m=0}^{n} \Phi\left(K_{d, t}, K_{s, t}, K_{c, t} \mid m\right) \cdot \Omega(m \mid n) \\
& \simeq \underset{n \in \mathbb{Z}^{+}}{\arg \max } \sum_{m=0}^{n} \Phi\left(K_{d, t}, K_{s, t}, K_{c, t} \mid m\right) \cdot \Lambda(m \mid n) \\
& =\underset{n \in \mathbb{Z}^{+}}{\arg \max } \Phi\left(K_{d, t}, K_{s, t}, K_{c, t} \mid n p_{t-1}^{\mathrm{ML}}\right) .
\end{aligned}
$$

Based on (15), the proposed AMLE-ALOHA scheme is given by

$$
\hat{p}_{t}=\min \left\{1, \frac{K}{\hat{N}_{t}}\right\}
$$

where

$$
\begin{aligned}
& \hat{N}_{t}=\frac{\hat{m}_{t}}{\hat{p}_{t-1}} \\
& \hat{m}_{t}=\underset{m \in \mathbb{Z}^{+}}{\arg \max } \Phi\left(K_{d, t}, K_{s, t}, K_{c, t} \mid m\right) .
\end{aligned}
$$

The operation procedure of the proposed scheme is summarized in Algorithm 1.

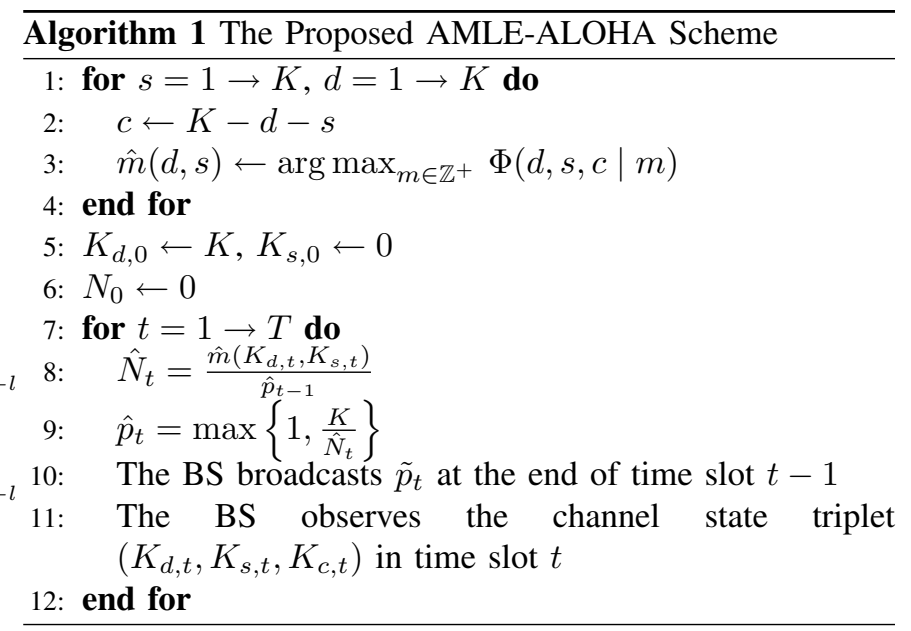




\section{Stability Analysis For AMLE-ALOHA}

The ALOHA protocol is an unstable protocol for large arrival rates [15]. In the unstable state, the system throughput becomes very low while users experience long delay. The maximum stable throughput $\lambda_{c}$ for multichannel slotted ALOHA with $K$ channels is given by $\lambda_{c}=K e^{-1}$ [13, Lemma 3]. We apply the drift analysis [16] to illustrate the stability of AMLE-ALOHA in the following.

The random process $X(t) \triangleq\left(N_{t}, \hat{N}_{t}\right)$ constitutes a twodimensional Markov chain. According to [17], the stability of AMLE-ALOHA is ensured if $X(t)$ is ergodic. We will show that $X(t)$ is ergodic using the stability theorems for two-dimensional process [13]. First, we need the following lemmas.

Lemma 1 [13]. The average drift of $N_{t}$ in the process $X(t)$ is

$E\left[N_{t}-N_{t-1} \mid N_{t}=n, \hat{N}_{t-1}=z\right]=\lambda-\frac{n K}{z}\left(1-\frac{1}{z}\right)^{n-1}$.

Lemma 2. If and only if the ML estimator $\hat{m}_{t}$ is consistent, we have

$$
\lim _{\sqrt{n^{2}+z^{2}} \rightarrow \infty} E\left[\hat{m}_{t} \mid N_{t}=n, \hat{N}_{t-1}=z\right]=\frac{n K}{z} .
$$

Proof. By definition of consistent ML estimator $\hat{m}_{t}$, we have

$$
\begin{aligned}
\lim _{\sqrt{n^{2}+z^{2} \rightarrow \infty}} & E\left[\hat{m}_{t} \mid N_{t}=n, \hat{N}_{t-1}=z\right] \\
& =E\left[m \mid N_{t}=n, \hat{N}_{t-1}=z\right] \\
& =n \hat{p}_{t-1} \\
& =\frac{n K}{z} .
\end{aligned}
$$

Then, we show that $X(t)$ is ergodic.

Theorem 3. If the ML estimator $\hat{m}_{t}$ is consistent and $n / z=1$, then $X(t)$ is ergodic and $\lambda_{c}=K e^{-1}$.

Proof. From Lemma 1, we have the average drift of $N_{t}$ in the process $X(t)$ given by

$$
\begin{aligned}
\mu_{n, z} & \triangleq \lim _{\sqrt{n^{2}+z^{2}} \rightarrow \infty, n / z=1} E\left[N_{t}-N_{t-1} \mid N_{t}=n, \tilde{N}_{t-1}=z\right] \\
& =\lambda-K e^{-1} .
\end{aligned}
$$

On the other hand, the average drift of $\hat{N}_{t}$ in the process $X(t)$ is

$$
\begin{aligned}
\nu_{n, z} & \triangleq \lim _{\sqrt{n^{2}+z^{2}} \rightarrow \infty, n / z=1} E\left[\hat{N}_{t}-\hat{N}_{t-1} \mid N_{t}=n, \hat{N}_{t-1}=z\right] \\
& \stackrel{(a)}{=} E\left[\frac{z \cdot \hat{m}_{t}}{K}-z \mid N_{t}=n, \hat{N}_{t-1}=z\right] \\
& \stackrel{(b)}{=} E\left[n-z \mid N_{t}=n, \hat{N}_{t-1}=z\right]=0
\end{aligned}
$$

where $(a)$ follows from (16) and (17), and (b) follows from Lemma 2. Thus, ergodicity holds for $\mu_{n, z}=\nu_{n, z}=0$ when $\lambda=\lambda_{c} \triangleq K e^{-1}$.

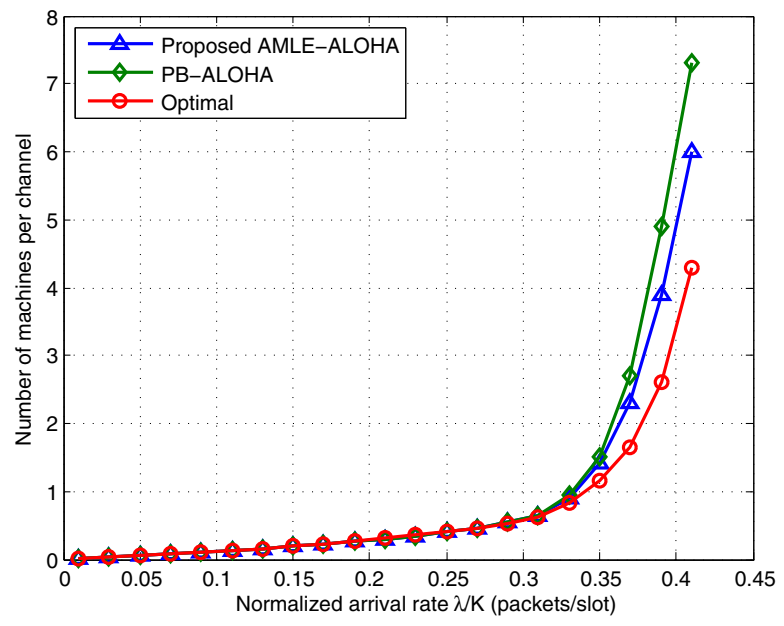

Fig. 3. Number of contending machines per channel vs. normalized packet arrival rate, with Poisson packet arrival and $K=5$ channels.

\section{NUMERICAL RESULTS}

In this section, we demonstrate the performance of the proposed AMLE-ALOHA scheme in comparison with the optimal multichannel control scheme and the PB-ALOHA scheme described in Sec. III. We set $K=5$ channels. We consider two cases of packet arrivals.

Case 1: Poisson packet arrivals: We consider that the packet arrivals follow a Poisson process, i.e.,

$$
\operatorname{Pr}\left(t, A_{t}=x\right)=f\left(A_{t}=x\right)=\frac{\lambda^{x} e^{-\lambda}}{x !}, \quad \forall t \in \mathbb{Z}^{+}
$$

where $E\left[A_{t}\right]=\lambda$ and $E\left[A_{t}^{2}\right]<\infty$. We run the simulation for $10^{6}$ time slots. It is assumed that there is an infinite number of machines so that no arrivals are discarded. Fig. 3 shows the number of contending machines per channel vs. the normalized packet arrival rate $\lambda / K$. Clearly, the number of active machines increases as $\lambda / K$ increases. Since active machines failing transmission will attempt transmission again in the next time slot, the system's backlogged machines accumulate as $\lambda / K$ increases. According to Theorem 3, the maximum stable throughput $\lambda_{c}=K e^{-1} \simeq 0.368 K$, and hence when $\lambda / K<0.34<e^{-1}=\lambda_{c} / K$, the system is in the stable region such that the number of contending machines per channel is smaller than or equal to 1 . When the system is stable, the performance of all three schemes is comparable; when the system becomes unstable, the proposed AMLE-ALOHA scheme has a smaller number of backlogged machines as compared to PB-ALOHA.

Fig. 4 shows the average packet transmission delay vs. the normalized packet arrival rate $\lambda / K$. Clearly, the packet transmission delay increases as $\lambda / K$ increases. It is observed that when $\lambda / K \leq 0.27$, almost all the packets can be transmitted in the next time slot with all three control schemes (i.e., the delay is less than two time slots). The proposed AMLE-ALOHA reduces the delay by about 3.2 time slots as compared to PB-ALOHA when $\lambda / K$ approaches the maximum stable rate 


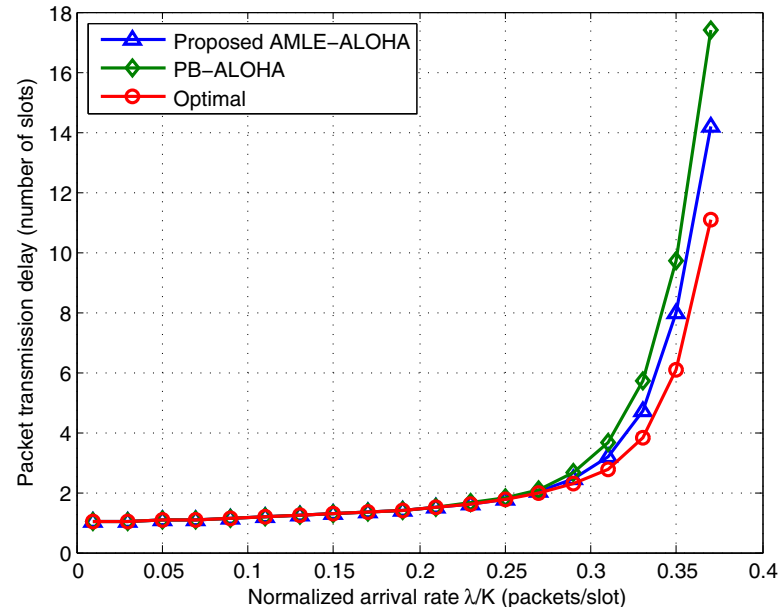

Fig. 4. Packet transmission delay vs. normalized packet arrival rate, with Poisson packet arrival and $K=5$ channels.

$\lambda_{c} / K \simeq 0.368$.

Case 2: Bursty packet arrivals: We examine the behavior of the control schemes under bursty traffic. This consideration represents many practical M2M communication scenarios where a large number of machines will suddenly be active in response to an emerging M2M application. We adopt the following packet arrival model for this scenario:

$$
A_{t}= \begin{cases}3000, & t=1 \\ 0, & t \geq 2, t \in \mathbb{Z}^{+}\end{cases}
$$

Fig. 5 shows the average percentage of backlogged machines vs. the elapsed time up to 2000 time slots. Initially, all the 3000 packets are awaiting transmission and thus there are 3000 backlogged machines in the system (100\%). As time elapses, an increasing number of packets are successfully transmitted and the number of backlogged machines decreases. It can be observed that PB-ALOHA and the proposed AMLEALOHA require 2000 and 1800 time slots, respectively, for transmitting all the 3000 packets (i.e., reaching the state of no backlogged machine). This is a $10 \%$ saving of reaction time by the proposed AMLE-ALOHA in a bursty traffic scenario.

\section{CONCLUSION}

We have studied the problem of congestion control based on slotted ALOHA for M2M communications. We proposed a new control scheme where the transmission probability of active machines is adapted according to the estimated number of current active machines in the system. We proposed an approximate ML estimation of the number of active machines based on the access outcomes in the previous time slot. We analytically examine the stability of the proposed control scheme using drift analysis. The proposed AMLEALOHA scheme was shown advantageous over the existing PB-ALOHA scheme both with partial information at the data aggregator, and comparable with the optimal control scheme with full information at the data aggregator.

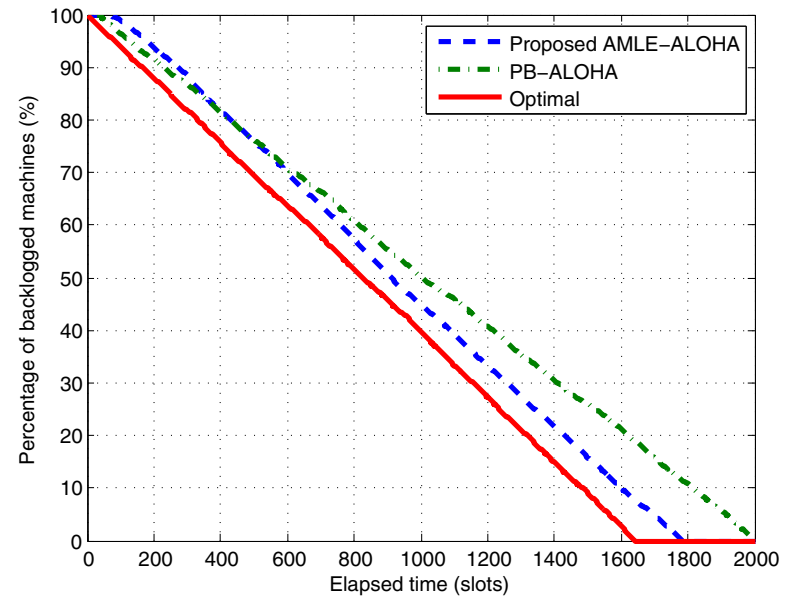

Fig. 5. Percentage of backlogged machines vs. elapsed time, with bursty packet arrival and $K=5$ channels.

\section{REFERENCES}

[1] A. Osseiran, V. Braun, T. Hidekazu, P. Marsch, H. Schotten, H. Tullberg, M. A. Uusitalo, and M. Schellmann, "The foundation of the mobile and wireless communications system for 2020 and beyond: Challenges, enablers and technology solutions," in Proc. IEEE VTC Spring, June 2013, pp. 1-5.

[2] C. Xie, K.-C. Chen, and X. Wang, "To hop or not to hop in massive machine-to-machine communications," in Proc. IEEE WCNC, Apr. 2013, pp. 1021-1026.

[3] M. Islam, A.-E. Taha, and S. Akl, "A survey of access management techniques in machine type communications," IEEE Commun. Mag., vol. 52, no. 4, pp. 74-81, April 2014.

[4] 3GPP, "Access class barring and overload protection," 3GPP TR23.898 V7.0.0, Mar. 2005.

[5] 3GPP, "Study on RAN improvements for machine-type communications," 3GPP TR37.868 V11.0.0, Sept. 2011.

[6] S.-Y. Lien, T.-H. Liau, C.-Y. Kao, and K.-C. Chen, "Cooperative access class barring for machine-to-machine communications," IEEE Trans. Wireless Commun., vol. 11, no. 1, pp. 27-32, January 2012.

[7] O. Arouk and A. Ksentini, "Multi-channel slotted aloha optimization for machine-type-communication," in Proc. ACM International Conference on Modeling, Analysis and Simulation of Wireless and Mobile Systems, Sep. 2014, pp. 119-125.

[8] L. G. Roberts, "Aloha packet system with and without slots and capture," SIGCOMM Comput. Commun. Rev., vol. 5, no. 2, pp. 28-42, Apr. 1975.

[9] W. Rosenkrantz and D. Towsley, "On the instability of slotted ALOHA multiaccess algorithm," IEEE Trans. Autom. Control, vol. 28, no. 10, pp. 994-996, Oct. 1983.

[10] R. L. Rivest, "Network control by Bayesian broadcast," IEEE Trans. Inf. Theory, vol. 33, no. 3, pp. 323-328, May 1987.

[11] J.-F. Frigon and V. C. M. Leung, "A Pseudo-Bayesian ALOHA algorithm with mixed priorities," Wirel. Netw., vol. 7, no. 1, pp. 55-63, Jan. 2001.

[12] H. Wu, C. Zhu, R. La, X. Liu, and Y. Zhang, "FASA: Accelerated SALOHA using access history for event-driven M2M communications," IEEE/ACM Trans. Networking, vol. 21, no. 6, pp. 1904-1917, Dec. 2013.

[13] O. Galinina, A. Turlikov, S. Andreev, and Y. Koucheryavy, "Stabilizing multi-channel slotted ALOHA for machine-type communications," in Proc. IEEE ISIT, July 2013, pp. 2119-2123.

[14] D. Shen and V. Li, "Performance analysis for a stabilized multi-channel slotted ALOHA algorithm," in Proc. IEEE PIMRC, vol. 1, Sept. 2003, pp. 249-253.

[15] D. Bertsekas and R. Gallager, Data Networks. Prentice-Hall, Inc., 1992.

[16] J. Tsitsiklis, "Analysis of a multiaccess control scheme," IEEE Trans. Autom. Control, vol. 32, no. 11, pp. 1017-1020, Nov. 1987.

[17] D. Chan and T. Berger, "Upper bound for the capacity of multiple access protocols on multipacket reception channels," in Proc. IEEE ISIT, July 2012, pp. 1603-1607. 